\title{
Organisms as Ecosystems/Ecosystems as Organisms
}

\author{
Minus van Baalen · Philippe Huneman
}

Published online: 21 October 2014

(c) Konrad Lorenz Institute for Evolution and Cognition Research 2014

It is well known that most organisms are composed of smaller subunits, including not only cells and semi-independent organelles but often also a whole suite of parasites, commensal organisms, and other symbionts (Buss 1987; Margulis 1970; Bouchard and Huneman 2013). The process of association of these subunits has been recognized as contributing to many of the major transitions that have occurred in the course of evolution (Maynard Smith and Szathmáry 1995; Michod 1999). As a result, the selective forces that have led to the subunits foregoing their evolutionary sovereignty are being better appreciated (van Baalen and Jansen 2001), but little is known yet about the mechanisms that maintain the dynamic coherence of the associations. In this respect there are many parallels with classical ecological questions. In spite of the fact that the processes that dynamically generate and maintain biodiversity in ecosystems (competition, herbivory, predation, parasitism, and so forth) are quite well known, we still have difficulty in understanding and predicting the outcome.

This parallel is obvious if one considers the history of the disciplines. Biologists and philosophers of biology have long

M. van Baalen ( $\square)$

Eco-Evolutionary Mathematics, Institut Biologie de l'Ecole Normale Supérieure (UMR 8197), CNRS, Paris, France

e-mail: minus.van.baalen@ens.fr

M. van Baalen

Eco-Evolutionary Mathematics, Institut Biologie de l'Ecole Normale Supérieure (UMR 8197), Ecole Normale Supérieure, Paris, France

\section{P. Huneman}

Institut d'Histoire et de Philosophie des Sciences et des Techniques (IHPST), CNRS/Université Paris I Sorbonne, Paris, France

e-mail: philippe.huneman@gmail.com been concerned with the status of associations of entities that are not stricto sensu organisms, but look like organisms at least as much as they look like collectives: ant colonies, wasp colonies (Seeley 1995; Hölldobler and Wilson 2008), termite mounds (Turner 2000), Portuguese men-of-war, slime molds (Bonner 1959), etc. The insight that organisms can be composed of other organisms is quite old, and dates back to the concept of "superorganism" already developed by the geologist Hutton in the 18th century. Since then, the idea has resurfaced multiple times, for instance, from the ecologist Clements (1916), following Forbes (1887) who suggested that lakes are microcosms, and more recently in Lovelock's Gaia hypothesis. Sober and Wilson (1998) reinitiated a discussion on precisely what superorganisms are (Bouchard 2010). This includes questions such as whether they are really targets of selection just like organisms (Gardner and Grafen 2009), and how they come into being (Reeve and Hölldobler 2007). Some evolutionary biologists argue, however, that the very concept of superorganism is unnecessary and can be replaced by a more general understanding of organism (Queller and Strassmann 2009).

Ecologists such as Clements were concerned by the fact that ecosystems (or at least some of them) seem to behave in a functionally unified and coherent way, with feedback loops, division of labor, regulations, and other features supposed to be characteristic of metazoan organisms. The notion of a "superorganism" thus carries at least implicitly a link with ecology. However, history proved to be on the other side-with those who, with Gleason (1926) and against Clements, argued that nothing special makes an ecosystem cohesive, and that the assemblage of species in a community does not reflect any kind of organicity. Ecological theory as it further unfolded in the 1950s and '60s (e.g., Hutchinson, MacArthur and Wilson, etc.) was built upon the notions of population, community, and ecosystem without using the concept of superorganism. 
However, the current state of our knowledge regarding ecosystems, and their stability, maintenance, and resilience bears many similarities with what we know about organisms and their adaptedness. Functional ecology has insisted on the fact that functional types could be distinguished in ecosystems (Simpson 1988; do Vale et al. 2010), so that differences of functional types may yield some division of labor as well as some differences in overall ecosystem outcomes such as resilience or productivity (Loreau and Hector 2001). Yet, even if we are capable of identifying most of the mechanisms that are involved, we are much less able to predict how ecosystems will respond when conditions change. In a similar fashion, it is not always obvious how the dynamic associations that are typical of organisms maintain their coherence and integrity in the face of many environmental challenges, as recent results in immunology or cancer biology can testify. For instance, the immune system is supposed to distinguish between self and non-self-many illnesses result from a failure of such distinction-but which criteria it should use is not obvious (Pradeu and Carosella 2006). A main reason for the failure of the immune system is that "self" is not a static entity but a dynamically changing set of epitopes (parts of molecules such as cell surface proteins and degraded virus particles that are recognized by the immune system). This dynamic view of organisms is emerging also in studies that address the evolutionary origins of cancer (Nagy 2004; Davila and Zamorano 2013), which includes the peculiar case of Tasmanian devil facial cancer transmitting itself from one individual to the next (McCallum and Jones 2006).

In this context it becomes appealing to postulate that both objects, organisms and ecosystems, would benefit from a common approach-in other words, to consider that to some extent ecosystems behave or function as some kind of organisms; or inversely, that organisms can be understood as kinds of ecosystems on a short timescale. This ecosystem view has been proposed by philosophers who stress that the genetic diversity within organisms (as including many varieties of symbionts, a whole microbiome, etc.) should compel us to see these individuals as multispecies associations (Dupré and O'Malley 2009; Bouchard and Huneman 2013). The insight that organisms may resemble ecosystems may benefit ecological theory too. In particular, our understanding of organismic cohesion, division of labor, or overall functionality, as well as the selective dynamics that occur at several levels (genes, cells, tissues), may help us to explain the emergence and stability of ecological communities along evolutionary timescales. Any ecosystem in the traditional, ecological sense (e.g., boreal forest, mangrove, wetland, etc.) is made of many kinds of species and abiotic elements, in which selection and physical dynamics take place at distinct levels and scales (Levin 1992). The extreme holistic or vitalist views such as those held by Clements, according to which an ecosystem would be a sort of large living being (a view that spans a long intellectual history, originating in the old Stoic view of the cosmos as an animal, and leading to versions of the Gaia hypothesis used in earth systems sciences; Lovelock and Margulis 1974) have been largely abandoned, but a weakened form of the ecosystem/organism analogy may still be fruitful in contemporary research, at least as a heuristic tool.

Indeed, many instances of this program can currently be found: some researchers investigate in a straightforwardly ecological way the composition and dynamics of the microbiome, explicitly "applying ecological theory" to the human microbiome (Costello et al. 2012), and more generally considering it as an ecosystem; others advocate an evolutionary view of cell dynamics that makes sense of cancer in a multilevel selection framework (Featherston and Durand 2012), in the way our understanding of diseases benefited from evolutionary approaches of host-parasite coevolution (evolution of virulence) (Alizon et al. 2009). Another research program uses ecological theory to study stem cell dynamics, through the concepts of niche and niche construction (Scadden 2006); and finally even the genome and its architecture, cohesion, and regulation within the cell can be addressed in the context of a "molecular ecology" (Raes and Bork 2008).

This thematic issue addresses questions and prospects related to the set of research programs that are fruitfully developing an analogy between ecosystems and organisms in either direction. The articles explore various parallels (and differences) between organismal coherence and ecological processes. Researchers explore either the ecological approach to understand organisms, or the individualizing behavior of many ecosystems as well as the criteria used to see them as individuals. Written from various standpoints (philosophy of biology, community ecology, evolutionary biology), the articles do not develop an overarching theory or framework but investigate different aspects of the set of research programs that could be loosely defined by the abovementioned analogy. They explore, among other things, the notions of ecological and biological individuality, the concept of fitness as applicable in ecological contexts, the connections between individuality and complexity as well as between information and individuality or adaptation. To this aim, they focus on case studies such as the immunity of Botryllus schlosseri, current research on the human gut microbiome, prospective gene therapy of a form of leukemia, different forms of symbiosis, or the use of complexity and entropy measures to capture differences between ecosystems.

This will hopefully create an opportunity for exploring the philosophical issues raised by this conceptual convergence. The present issue does not aim to be exhaustive or 
even systematic, but rather intends to illustrate the many parallels between the notions of organism and ecosystem.

It is now universally acknowledged that almost anything in biology is made up out of smaller subunits at a series of hierarchical levels. This holds for organisms (roughly, individuals-organs-tissues-cells-organelles-DNA) as well as for ecological entities (roughly, ecosystemshabitats-communities-species-populations-subpopulations-individuals) so it is tempting to explore the analogy between the two. In the two-part article, "Individuality as a Theoretical Scheme," Philippe Huneman explores the consequences of an abstract definition that extracts an individual from the complete set of interactions among a full set of basic entities. In effect, he proposes that one can argue that a set for which the within-set of interactions are more important than the external interactions acquires a degree of individuality. Since this still incorporates a level of fuzziness, Huneman calls this the weak definition of individuality, as opposed to a strong definition, which requires strong cohesion and integration, needed to allow individuals to become units of adaptation in their own right. (A "unit of adaptation" is that which benefits from a given adaptation; often the term "unit of selection" is used in this respect, but this term can be confusing as it has acquired many other meanings; van Baalen and Jansen 2001.)

However, even not fully integrated individuals ("weak" individuals, in Huneman's sense) can act as units of adaptation. The consequences of this view are explored in "Ecosystem Evolution is about Variation and Persistence, not Populations and Reproduction" by Frédéric Bouchard. Considering in some detail how termites form associations with the fungi upon which they depend for the digestion of the cellulose-rich materials they forage for, Bouchard shows that two cases that evolutionary biologists would consider as quintessentially different do nevertheless function and evolve in very similar ways.

"Methodology and Ontology in Microbiome Research" by John Huss provides another perspective on how individuals actually depend on a whole suite of symbiotic organisms. Huss describes attempts to characterize the state and dynamics of what has come to be termed the microbiome, i.e., the ensemble of genetic information of the host and its suite of symbionts. As he discusses, there were some exciting early reports that chimpanzees and humans have one of three possible characteristic ensembles of intestinal flora, but it appears that the actual situation is less clear-cut. It is therefore essential that the study of microbiomes incorporate the fact that these are highly dynamic and variable associations without precise characteristics. Yet at the same time an ungulate would not be able to survive and reproduce if these associations were completely random either.
In the two-part "Ecological Models for Gene Therapy," Arnaud Pocheville, Maël Montévil, and Régis Férrière try to go one step further towards an ecological view of organisms, and study how insights into the associative nature of individuals can be used to develop strategies for medical intervention. They rely on the assumption that mutant cell lineages may be governed not so much by a density-dependent growth rate, but rather by a form of density-dependent inertial dynamics (conditions may determine whether growth accelerates or decelerates rather than dictate their absolute values). Given such an assumption, the success of various grafting strategies (as practiced in genetic therapy) can be predicted.

In "Allorecognition, Germline Chimerism, and Stem Cell Parasitism in the Colonial Ascidian, Botryllus schlosseri," the relationship between individuality and the fate of grafts is also addressed by Tony De Tomaso, who studies the role of allorecognition in a colonial organism. It is often thought that allorecognition is closely linked to the functioning of an immune system, but De Tomaso shows that a Botryllus species-an aquatic ascidian-has an MHC-like recognition system even though it has no immune system. This is no surprise, however, once it is recognized that there are large evolutionary advantages for a sessile colonial organism that can detect whether other units it comes into contact with are part of the same or of another, competing colony.

In "Adaptation, Conflicting Information, and Stress," Minus van Baalen explores some of the consequences of the fact that members of compound individuals need to communicate (exchange information) in order to coordinate their activities. This is fine for a monolithic individual, but it is more than likely that members of a compound individual do not have fully overlapping evolutionary interests, which reduces the benefits of adhering to a single communication standard. Members of an association then have to assess the various cues they perceive, which may lead to a form of information-induced stress as different cues may be in conflict.

In "Ecosystem Complexity through the Lens of Logical Depth: Capturing Ecosystem Individuality," a very different approach is adopted by Cédric Gaucherel, who tries to assess the complexity of ecosystems by applying notions adopted from information and complexity theories. For instance, logical depth is a complexity measure that takes into account the time of the shortest computer program that mimics the real system. Using a range of Gaia-style Daisyworlds that differ in complexity, Gaucherel finds that simulations of the more complex ones indeed need more time to converge. This approach provides a starting point for developing more formal measures of degrees of individuality. 
In the last article, "Group Selection and Group Adaptation During a Major Evolutionary Transition: Insights from the Evolution of Multicellularity in the Volvocine Algae," Deborah Shelton and Rick Michod address the fundamental issue that observing groups adapting to some environmental condition does not necessarily imply the action of group selection-it may just be a side effect of the individual members adapting to these conditions. In particular, if we want to assess whether ecosystems acquire a form of individuality it is important to distinguish true group selection from such "counterfactual" group adaptation.

\section{References}

Alizon S, Hurford A, Mideo N et al (2009) Virulence evolution and the trade-off hypothesis: history, current state of affairs and the future. J Evol Biol 22:245-259

Bonner JT (1959) The cellular slime molds. Princeton University Press, Princeton

Bouchard F (2010) Symbiosis, lateral function transfer and the (many) saplings of life. Biol Philos 25:623-641

Bouchard F, Huneman P (eds) (2013) From groups to individuals. MIT Press, Cambridge

Buss LW (1987) The evolution of individuality. Princeton University Press, Princeton

Clements FE (1916) Plant succession: an analysis of the development of vegetation. Carnegie Institution of Washington, Washington, DC

Costello E, Stagaman K, Dethlefsen L et al (2012) The application of ecological theory toward an understanding of the human microbiome. Science 336:1255-1262

Davila AF, Zamorano P (2013) Mitochondria and the evolutionary roots of cancer. Phys Biol 10:026008

do Vale VS, Schiavini I, de Oliveira AP et al (2010) When ecological functions are more important than richness: a conservation approach. J Ecol Nat Environ 2:270-280

Dupré J, O’Malley MA (2009) Varieties of living things: life at the intersection of lineage and metabolism. Philos Theory Biol 1:e003

Featherston J, Durand PM (2012) Cooperation and conflict in cancer: an evolutionary perspective. S Afr J Sci 108(9/10):1-5
Forbes G (1887) The lake as a microcosm. Bull Sci Assoc Peoria, IL):77-87

Gardner A, Grafen A (2009) Capturing the superorganism: a formal theory of group adaptation. J Evol Biol 22:659-671

Gleason HA (1926) The individualistic concept of the plant association. Bull Torrey Bot Club 53:7-26

Hölldobler B, Wilson EO (2008) The superorganism: the beauty, elegance, and strangeness of insect societies. Norton, New York

Levin SA (1992) The problem of pattern and scale in ecology. Ecology 73:1943-1967

Loreau M, Hector A (2001) Partitioning selection and complementarity in biodiversity experiments. Nature 412:72-76

Lovelock J, Margulis L (1974) Atmospheric homeostasis by and for biosphere-Gaia hypothesis. Tellus 26:2-10

Margulis L (1970) Origin of eukaryotic cells. Yale University Press, New Haven

Maynard Smith J, Szathmáry E (1995) The major transitions in evolution. Oxford University Press, Oxford

McCallum H, Jones M (2006) To lose both would look like carelessness: Tasmanian devil facial tumour disease. PLoS Biol 4(10):e342-e432

Michod RE (1999) Darwinian dynamics. Princeton University Press, Princeton

Nagy JD (2004) Competition and natural selection in a mathematical model of cancer. Bull Math Biol 66:663-687

Pradeu T, Carosella ED (2006) On the definition of a criterion of immunogenicity. Proc Natl Acad Sci USA 103:17858-17861

Queller DC, Strassmann JE (2009) Beyond society: the evolution of organismality. Phil Trans R Soc Lond B 364:3143-3155

Raes J, Bork P (2008) Molecular eco-systems biology: towards an understanding of community function. Nat Rev Microbiol 6:693-699

Reeve HK, Hölldobler B (2007) The emergence of a superorganism through intergroup competition. Proc Natl Acad Sci USA 104:9736-9740

Scadden D (2006) The stem-cell niche as an entity of action. Nature 441(7097):1075-1079

Seeley TD (1995) The wisdom of the hive: the social physiology of honey bee colonies. Harvard University Press, Cambridge

Simpson BB (1988) Biological diversity in the context of ecosystem structure and function. Biol Int 17:15-17

Sober E, Wilson DS (1998) Unto others: the evolution and psychology of unselfish behavior. Harvard University Press, Cambridge

Turner JS (2000) The extended organism: the physiology of animalbuilt structures. Harvard University Press, Cambridge

van Baalen M, Jansen VAA (2001) Dangerous liaisons: the ecology of private interest and common good. Oikos 95:211-224 